\title{
The contribution of X-ray polar blowout jets to the solar wind mass and energy
}

\author{
Giannina Poletto ${ }^{1}$, Alphonse C. Sterling ${ }^{2}$, Stefano Pucci ${ }^{3}$ and \\ Marco Romoli ${ }^{3}$ \\ ${ }^{1}$ INAF - Arcetri Astrophysical Observatory, \\ Largo Fermi, 5, 50125, Firenze, Italy \\ email: poletto@arcetri.astro.it \\ ${ }^{2}$ Space Science office, VP 62, MSSC, \\ Huntsville, AL 35812, USA \\ email: alphonse.sterling@nasa.gov \\ ${ }^{3}$ University of Firenze, Firenze, Italy \\ email: stpucci@arcetri.astro.it \\ ${ }^{4}$ University of Firenze, Firenze, Italy \\ email: mromoli@unifi.it
}

\begin{abstract}
Blowout jets constitute about $50 \%$ of the total number of X-ray jets observed in polar coronal holes. In these events, the base magnetic loop is supposed to blow open in what is a scaled-down representation of two-ribbon flares that accompany major coronal mass ejections (CMEs): indeed, miniature CMEs resulting from blowout jets have been observed. This raises the question of the possible contribution of this class of events to the solar wind mass and energy flux. Here we make a first crude evaluation of the mass contributed to the wind and of the energy budget of the jets and related miniature CMEs, under the assumption that small-scale events behave as their large-scale analogs. This hypothesis allows us to adopt the same relationship between jets and miniature-CME parameters that have been shown to hold in the larger-scale events, thus inferring the values of the mass and kinetic energy of the miniature CMEs, currently not available from observations. We conclude our work estimating the mass flux and the energy budget of a blowout jet, and giving a crude evaluation of the role possibly played by these events in supplying the mass and energy that feeds the solar wind.
\end{abstract}

Keywords. Sun: activity, Sun: solar wind, Sun: Coronal Mass Ejections.

\section{Introduction}

As a result of the analysis of HINODE data, the estimated number of X-ray polar jets has been soaring from a few per day to an average of 10 per hour (Cirtain et al. 2007). Successively, it became clear that jets may be classified either as "standard", or as "blowout" events, depending on their characteristics (Moore et al. 2010). Standard jets are suspected to arise from reconnection between an emerging bipolar arch and the open unipolar ambient field of the polar coronal hole regions. In blowout jets, it is suggested that an emerged arch, similar to that of standard jets, becomes unstable, erupts, and blows open as it reconnects with the open ambient field. That erupting arch can contain a cool filament that becomes a cool jet mimicking on a miniature scale what happens in large-scale coronal mass ejections (CMEs). Fig. 1 shows a typical polar coronal hole, as seen in the HINODE/XRT Al/Poly filter on March 7, 2008, where a narrow pencil-like jet is clearly seen together with other smaller structures.

After Moore et al. (2010) paper, a number of authors presented further evidence of the occurrence of two classes of jets and even detected the mini CMEs associated with 


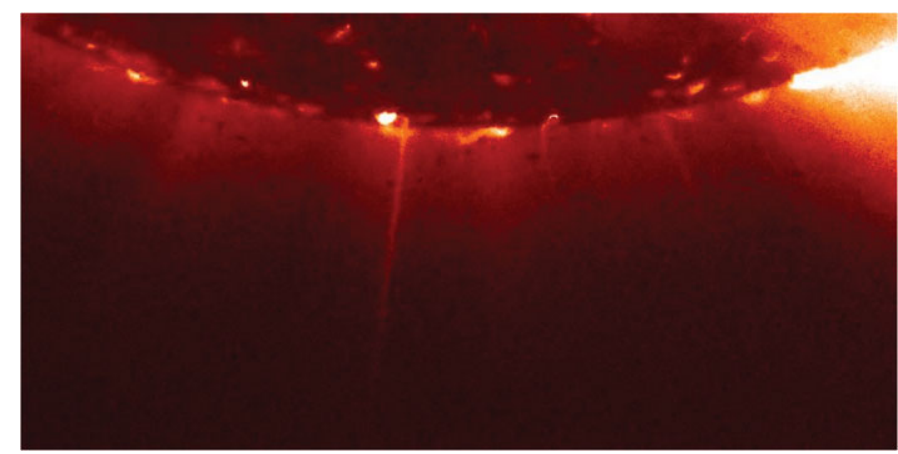

Figure 1. HINODE XRT image of the southern polar region acquired in the Al/Poly filter on March 7, 2008, at 08:43:48 UT, showing a pencil like jet and other smaller features rooted in the coronal hole area [Courtesy of P. Grigis].

blowouts (Hong et al. 2011, Shen et al. 2012), although the CME masses could not be measured. Analogous to the situation of confined vs. eruptive flares, blowout jets have a longer duration and are more energetic as compared to the standard jets Pucci et al. (2013). Observations in polar coronal holes reveal about an equal number of standard and blowouts (Moore et al. 2013).

Nowadays the properties of standard and blowout jets seem to be, morphologically, thoroughly defined, but their physical characteristics have been scarcely explored. Knowledge of the density, temperature, outflow speed of the jets is relevant per se, to understand the behavior of this miniature class of events with respect to their large-scale counterparts, and because it allows us to check whether jets represent the long-searched source of solar wind mass and energy. Here we adopt the physical parameters that have been inferred, from an analysis of typical ejections, by Pucci et al. (2013) and, on this basis, we calculate the energy budget of jets, focusing on a blowout jet. Blowout jets are more energetic than standard jets, and so if they turn out to be inadequate to supply the solar wind mass and energy, then standard jets will be ineffective in this regard as well. In the next section, we summarize the jet parameters and then proceed to calculate their energy budget. In Section 3, we evaluate the energy associated with the cool component of blowouts and give an estimate of the mini-CME mass. We conclude discussing the jet mass flux and energy in the context of the solar wind mass and energy requirements.

\section{The physical parameters of jets and their energy budget}

Spectroscopic techniques allow us to infer the physical parameters of jets seen in different X-ray bands by different experiments/spacecraft. Pucci et al. (2013) used data from HINODE/XRT and STEREO/SECCHI experiments to evaluate temperature, density and outflow speed of standard and blowouts. They found that, for a standard jet, the electron temperatures $\left(T_{e}=1.6 \times 10^{6} \mathrm{~K}\right)$ was lower by $\approx 15 \%$, densities $\left(6 . \times 10^{8} \mathrm{~cm}^{-3}\right)$ were about the same and outflow speeds $\left(\approx 250 \mathrm{kms}^{-1}\right)$ were lower by $\approx 40 \%$ than in the blowout jet. Because blowouts, which are composed of multiple structures, last longer than standard jets, the role of the latter, if any, in contributing to the wind mass and energy is minor, and justifies our previous statement that blowouts are the most energetic representatives of the jets family. Hence, from here on, we focus on this class of jets.

As mentioned earlier, reconnection is crucial in the origin and development of jets. The magnetic energy flux delivered in reconnection episodes is partitioned among enthalpy, 
wave, kinetic, potential and radiative energy fluxes of the ensuing jets. Because temperature was approximately constant along the axes of the jets studied in Pucci et al. (2013), we ignore the conductive energy flux and we write the above terms as

$$
\begin{gathered}
F_{\text {enth }}=\frac{\gamma}{\gamma-1} p v \\
\left.F_{w}=\sqrt{(} \frac{\rho}{4 \pi}\right) \xi^{2} B \\
F_{\text {kin }}=1 / 2 \rho v^{3} \\
F_{\text {pot }}=\rho g L v \\
F_{\text {rad }}=n_{e} n_{H} \chi T^{\alpha} L
\end{gathered}
$$

where $p, \rho, g, \gamma$ are, respectively, the plasma pressure, mass density, gravity, $\gamma$ is the ratio of the specific heats $(\gamma=5 / 3), n_{e}$ is the plasma number density $\left(n_{H}=n_{e}\right), \xi$ is the amplitude of unresolved non-thermal plasma motion, $\mathrm{B}$ is the field strength and $\mathrm{L}$ is the jet length. The parameters $\chi$ and $\alpha$ appear in the analytical approximation to $F_{\text {rad }}$ given by Rosner et al. (1978). The most uncertain energy flux is $F_{w}$ : lacking direct evidence of the amplitude of Alfvénic waves excited by reconnection, we assume that waves show up only as unresolved plasma motions and adopt a wave amplitude of 100 $k m s^{-1}$ in agreement with values found by Kim et al. (2007) from EIS observations of line broadening in jets. The field strength B has been derived from the assumption that outflows occur at the Alfvén speed. An evaluation of the above stated energy fluxes leads to a blowout total energy of $\approx 2 \times 10^{27} \mathrm{erg}$. Hence blowouts are typically $10^{-5}-10^{-6}$ times less energetic than the largest flares associated with CMEs. Pucci et al. (2013) provide more details of these calculations.

\section{The cool component of blowouts: the associated CME and its energy}

When calculating the energy budget of blowouts, we should include also the cool component associated with the event: in their large-scale counterparts the kinetic/potential energy associated with the ejecta plays a major role (Emslie et al. 2012). Although coolmaterial outflows have been detected above the limb by white light coronagraphs, the amount is usually too tiny to be estimated via conventional methods. On the other hand, knowledge of the CME mass is a prerequisite for calculating the CME's energy; we resorted to an indirect technique for estimating the mass, and subsequently the energy, by assuming blowout jets behave as their large-scale counterparts.

Yashiro \& Gopalswamy (2009) have shown that there is a relationship between the 1-8 $\AA$ X-ray flare fluence and the kinetic energy of the corresponding CME. Analogously Aarnio et al. (2011) have shown that the flare X-ray flux is correlated with the mass of the associated CME. Hence, we estimated the X-ray emission of the blowout from its density and temperature (which can be easily done either analytically or via standard codes like CHIANTI) and inferred the CME mass and its kinetic energy, via the relationships given by the above authors. It turns out that the expected CME kinetic energy is of the order of a few times $10^{26} \mathrm{erg}$ and its mass of the order of $10^{12} \mathrm{~g}$. Although these estimates are quite crude, they are based on extrapolations to small-scale events of well established relations for larger-scale events. We conclude that the energy residing in the cool component of blowouts is of the same order as that of the hot component. 


\section{Jets and the solar wind}

We now discuss our results in terms of the relevance of jets as contributors to the solar wind mass and energy flux. The mass flux $n v$ originating from the ejections can be estimated from the density and outflow speed values given above. We limit our analysis to blowouts whose occurrence rate is still not precisely known, but we assume here 50 events per day. Assuming a base radius of the order of $1000 \mathrm{~km}$, for the bright components of the blowout and an event duration of the order of $20 \mathrm{~min}$, it turns out that blowouts are the source of a mass flux of $5 \times 10^{5} \mathrm{~cm}^{-2} \mathrm{~s}^{-1}$, which is smaller than typical wind mass flux $\left(\approx 2 \times 10^{8} \mathrm{~cm}^{-2} \mathrm{~s}^{-1}\right)$ by about a factor of $10^{3}$. This estimate is affected by large uncertainties: however, even if the total number of jets, their outflows, their base area, were each underestimated by a factor 3 (which is unlikely) jets could supply only a few hundredths of the wind mass flux.

We point out that we neglected the contributions that mini CMEs might supply to the wind. If it would be proven that each blowout is accompanied by a mini-CME, we might hypothesize that this is a further source of mass flux. However, a quick order-ofmagnitude estimate shows that even if this were the case, a rate of 50 small CMEs per day cannot contribute appreciably to the wind mass flux.

The wind energy flux $F_{\text {wind }}$ is of the order of $10^{5} \mathrm{erg} \mathrm{cm}^{-2} \mathrm{~s}^{-1}$ (see, e.g. Le Chat, 2012 and Schwenn, 2006). We have shown that the total energy released by blowouts is on the order of a few units times $10^{27}$ erg per event. Taking into account their total number per day and their duration, we end up with an energy flux of $\approx 10^{-4} F_{\text {wind }}$, much too small to be accounted for by uncertainties in our calculations. If we underestimated the value of $\mathrm{B}$ - which is likely, as bulk flows may not be as fast as small-scale reconnection flows - the wave flux $F_{w}$ will also be underestimated. Analogously, outflow speeds may have been underestimated, leading to low values of the kinetic flux $F_{k i n}$. Nevertheless, our estimates cannot be off by more than a factor of 100 , which is insufficient to make blowouts contribute appreciably to the wind. We conclude that blowouts cannot provide for the mass and energy of solar wind. However, we did not include other features, possibly associated with blowouts, nor we counted type II spicules as blowouts. If the latter turn out to be part of the same class of events, our conclusion may need to be revised.

GP acknowledges support from ASI I/015/07/0. ACS was supported by funding from NASA's Office of Space Science through the Living with a Star Targeted Research and Technology Programs.

\section{References}

Aarnio, A. N., Stassun, K. G., Hughes, W. J., \& McGregor, S. L. 2011, SP, 268, 195

Cirtain, J. W., Golub, L., Lundquist, A., van Ballegoijen, Savcheva, A., et al. 2007, Science, 318,1580

Emslie, A. G., Dennis, B. R., Shin, A. Y., et al. 2012, ApJ, 759, 71

Hong, J., Jiang, Y., Zheng, R., Yang, J., Bi, Y., Zinner, E., \& Yang, B. 2011, ApJ (Letters), $738, \mathrm{~L} 20$

Kim, Y. H., Moon, Y.-J., Park, Y.-D., Sakurai, T., Chae, J. et al. 2007, PASJ, 59, S763

Le Chat, G., Issautier, K., \& Meyer-Vernet, N. 2012, SP, 279, 197

Moore, R. L., Cirtain, J. W., Sterling, A. C., \& Falconer, D. A. 2010, ApJ, 720, 757

Moore, R. L., Cirtain, J. W., Sterling, A. C., Falconer, D. A., \& Robe, D. 2013, ApJ, 769, 134

Pucci, S., Poletto, G., Sterling, A. C., \& Romoli, M. 2013, ApJ, 776, 16

Rosner, R., Tucker, W. H., \& Vaiana, G. S. 1978, ApJ, 220, 643

Schwenn, R. 2006, SSR, 124, 51

Shen, Y., Liu, Y., Su, J., \& Deng, J. 2012, ApJ, 745, 164

Yashiro, S. \& Gopalswamy, N. 2009, IAU Symp., 17, 233 